\title{
Physiological and transcriptomic characterization of a yellow- green leaf mutant of maize
}

\author{
Tingchun Li \\ Anhui Academy of Agricultural Sciences \\ Huaying Yang \\ Anhui Academy of Agricultural Sciences \\ Yan Lu \\ Western Michigan University \\ Qing Dong \\ Anhui Academy of Agricultural Sciences \\ Guihu Liu \\ Anhui Academy of Agricultural Sciences \\ Feng Chen \\ University of Tennessee \\ Yingbing Zhou (D 54189341@qq.com) \\ Anhui Academy of Agricultural Sciences
}

\section{Research article}

Keywords: Yellow-green leaf; Chlorophyll biosynthesis; Photosynthesis; Tricarboxylic acid cycle; Secondary metabolism; Transcriptome analysis

Posted Date: May 1st, 2020

DOI: https://doi.org/10.21203/rs.3.rs-24848/v1

License: @ (i) This work is licensed under a Creative Commons Attribution 4.0 International License. Read Full License 


\section{Abstract}

Background Chlorophylls, green pigments in chloroplasts, are essential for photosynthesis. Reduction in chlorophyll contents may result in retarded growth, dwarfism, and sterility. In this study, a yellow-green leaf mutant of maize, indicative of abnormity in chlorophyll contents, was identified. The physiological parameters of this mutant were measured. Next, global gene expression of this mutant was determined using transcriptome analysis and compared to that of wild-type maize plants. Results The yellow-green leaf mutant of maize was found to contain lower contents of chlorophyll a , chlorophyll b and carotenoid compounds. It contained fewer active PSII centers and displayed lower values of original chlorophyll fluorescence parameters than the wild-type plants. The real-time fluorescence yield, the electron transport rate, and the net photosynthetic rate of the mutant plants showed reduction as well. In contrast, the maximum photochemical quantum yield of PSII of the mutant plants was similar to that of the wild-type plants. Comparative transcriptomic analysis of the mutant plants and wildtype plants led to the identification of differentially expressed1122 genes, of which 536 genes were up-regulated and 586 genes down-regulated in the mutant. Five genes in chlorophyll metabolism pathway, nine genes in the tricarboxylic acid cycle and seven genes related to the conversion of sucrose to starch displayed down-regulated expression. In contrast, genes encoding a photosystem II reaction center PsbP family protein and the PGR5-like protein 1A (PGRL1A) exhibited increased transcript abundance. Conclusions The yellow-green leaf mutant of maize contained fewer active PSIl centers with lowered net photosynthesis rate, but have the similar value of the maximum photochemical quantum yield of PSII with that of the wild-type plants. Analysis of differentially expressed genes through transcriptome analysis revealed the down-regulated genes which may be responsible for chlorophyll deduction and changes of photosynthetic characteristics. The up-regulated genes would be helpful to maintain the active PSII centers of the yellow-green leaf mutant.

\section{Background}

Chlorophylls are essential pigments for photosynthesis, playing the main role in the conversion of light energy to stored chemical energy [1]. Chlorophyll contents directly determine photosynthetic potential and primary productivity of green plants[13].The formation of chlorophyll consists of four steps including synthesis of 5-aminolevulinic acid, formation of a pyrrole ring porphobilinogen, synthesis of protoporphyrin IX and insertion of $\mathrm{Mg}^{2+}$ to the protoporphyrin IX[4]. The functional genes of the chlorophyll metabolism pathway have been identified.

Generally, the leaf color is green for its common content of chlorophyll. Nevertheless, a large number of leaf color mutants have been identified in many seed plant species, such as Arabidopsis, maize, soybean, barley, rice, and wheat [5]. Among the leaf color mutants, a number of abnormal phenotypes have been identified, such as yellow, pale green, spots, and stripes. Due to reduced levels of chlorophyll, retarded growth, dwarfism, and sterility were characterized in most color mutants. Recently, with the progressive characterization of various leaf color mutants, a significant number of genes have been isolated and verified to be responsible for the abnormal phenotype. For example, in carrot, a YEL locus was mapped in a linkage group with a total length of $33.2 \mathrm{cM}$, and the mutant had a yellow-leaf phenotype [6]. In maize, a semi-dominant oil yellow 1 (OyI) mutant was identified to be deficient in the conversion of protoporphyrin IX to magnesium protoporphyrin IX [7]. The Oy/gene was

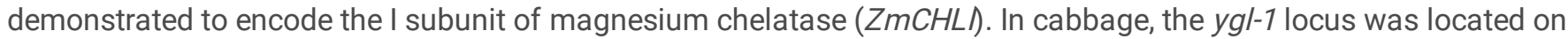
chromosome C01; mutation in the $\mathrm{ygl-1gene} \mathrm{exhibited} \mathrm{a} \mathrm{yellow-green} \mathrm{leaf} \mathrm{phenotype} \mathrm{[8].} \mathrm{In} \mathrm{rice,} \mathrm{mutation} \mathrm{in} \mathrm{the} \mathrm{VIRESCENT}$ YELLOW LEAF $(V Y L)$ gene, which encodes a subunit of chloroplast Clp (OsClpP6), resulted in temperature-insensitive and developmental stage-dependent virescent yellow leaf ( $\mathrm{vyl}$ ) phenotype [9]. $\mathrm{Fg} /$ is located in the coding region of OsPORB, and its mutation resulted in the presence of the yellow/white leaf [10]. Mutation in the $F d C 2$ gene, which encodes a ferredoxin-like protein with a C-terminal extension, caused the yellow-green leaf phenotype in rice [11]. Mutation in the rice YS83 (LOC_Os02g05890) gene resulted in the yellow-green-leaf phenotype as well [12]. In addition, a number of chloroplast signal recognition particle (cpSRP) mutants were identified with chlorophyll deficiency in Arabidopsis, rice and maize [13-15]. Although these studies have provided many insights into the key genes controlling chlorophyll deficiency, the analyses of physiological parameters of such mutants and their underlying molecular mechanisms have been generally lacking.

Chlorophyll-deficient mutants are important tools for studying the formation and development of photosynthetic pigments in plants. Their phenotypes could be used as crop trait markers in hybrid breeding [16]. In this work, a new yellow-green leaf 
mutant inbred line of maize was isolated. The chlorophyll contents, chlorophyll fluorescence parameters, and photosynthesis characteristics were determined. Using comparative transcriptomic analysis, differentially expressed genes were compared between the yellow-green leaf mutant and the normal green leaf inbred line. These results not only provide valuable genetic resources for further studies of chlorophyll-deficient mutants in maize, but also contribute to our understanding of the relationship between physiological changes and gene expression changes. The latter may pave the way to further dissecting the molecular basis of morphological and physiological characteristics of the yellow-green leaf mutant.

\section{Results}

\section{Identification of a maize yellow-green leaf mutant and measurement of its pigment contents}

A yellow-green leaf mutant inbred line was firstly isolated from an F2 segregating population of the cross recombination inbred line $\mathrm{C033}$ and inbred line LH102, both of which have a green leaf phenotype. After successive self-pollination of F2, F3 and F4 generations, a stable F5 generation was obtained. In this study, a stable yellow-green leaf mutant inbred line and a normal green leaf inbred line from the F5 generation were selected for downstream characterizations. The yellow-green leaf mutant had a yellow color in the entire above-ground portion of the plant (Fig. 1A).

Leaf color could indicate the amount and proportion of chlorophyll in leaves. Deficiency of chlorophyll leads to the leaf color change from green to yellow. In this study, in contrast with the normal green leaf inbred line, the content of chlorophyll $a$ and chlorophyll $b$ in the yellow-green leaf mutant was reduced by $35.22 \%$ and $34.48 \%$, respectively, which may directly result in the presence of yellow-green color in the mutant plants (Fig. 1B). Otherwise, the contents of seven kinds of carotenoid compounds including neoxanthin, violaxanthin, capsanthin, zeaxanthin, [-carotene, $\beta$-carotene and lutein were significantly decreased in the yellow-green leaf mutant (Fig .1C).

\section{Measurement of chlorophyll fluorescence parameters of the yellow-green leaf mutant}

For further analysis, chlorophyll fluorescence parameters were determined to evaluate the changes of light absorption and energy transfer in the light-harvesting complexes. Fo indicates the minimum fluorescence yield after dark-adaptation with all PSIl centers open. Fm represents the maximum fluorescence yield after dark-adaptation with all PSIl centers closed. Both Fo and Fm were decreased in the yellow-green leaf mutant, suggesting that the yellow-green leaf mutant has fewer active PSII centers than the normal green leaf inbred line (Fig. 2A). However, the yellow-green leaf mutant and the normal green leaf inbred line had the similar value of the maximum photochemical quantum yield of PSII (Fv/Fm), suggesting that light absorption and energy transfer of the light-harvesting complexes is still efficient in the yellow-green leaf mutant plants.

Ft is the real-time fluorescence yield recorded during the slow kinetics induction with the continuous monitoring mode. The changes of Ft reflect the light-adaption status of the PSII centers. Although the Ft kinetics curve in the yellow-green leaf mutant had the same light-adaption pattern as that in the normal green leaf inbred line, the Ft values were much lower in the yellowgreen leaf mutant (Fig. 2B). Accordingly, the values of the minimal and maximal fluorescence yield in the light-adapted state (Fo' and Fm') were significantly lower in the yellow-green leaf mutant than in the normal green leaf inbred line (Fig.2C).

Photochemical quenching parameters ( $\Phi_{\mathrm{PSI}}, \mathrm{qP}$, and $\left.\mathrm{qL}\right)$, non-photochemical quenching parameters $\left(\Phi_{\mathrm{NO}}, \Phi_{\mathrm{NPQ}}, \mathrm{NPQ}\right.$, and $\left.\mathrm{qN}\right)$, and the PSIl electron transport rate (ETR) were also evaluated. The values of $\Phi_{\mathrm{PSN}}, \mathrm{qP}, \mathrm{qL}, \Phi_{\mathrm{NO}}, \Phi_{\mathrm{NPQ}}, \mathrm{NPQ}$, and qN were similar between the yellow-green leaf mutant and the normal green leaf inbred line (Fig. 2C). But the value of ETR was significantly lowered in the yellow-green leaf mutant.

\section{Net photosynthesis in response to light intensities of the yellow-green leaf mutant}

Net photosynthesis (Pn) in response to different light intensities was also determined (Fig.3). Pn was lower in the yellow-green leaf mutant than in the normal green leaf inbred line when the light intensity was $1200 \mu \mathrm{mol}$ photonsm ${ }^{-2} \mathrm{~s}^{-1}$ or higher. But the rate of dark respiration was higher in the yellow-green leaf mutant.

Comparative transcriptomic analysis of the yellow-green leaf mutant and the normal green leaf inbred line: overall changes

Page $3 / 21$ 
To explain the physiological changes in the yellow-green leaf mutant, comparative transcriptome analysis was performed to identify differentially expressed genes in chlorophyll biosynthesis, light-harvesting antenna complex formation, photosynthesis, and other metabolism pathways. Three independently repeated sequencing of cDNA was carried out by using the HiSeq 2500 platform. After data processing, the clean reads were obtained. Pearson correlation was calculated for the three independent experiments. The data from NGL_1, NGL_3, YGL_2, and YGL_3 were collected for the following analysis for the higher correlation rate between the same color leaves (Additional file: Fig. S1). Further studies were performed to identify differentially expressed genes, using DGE methods. A total of 1122 genes were found to be differentially expressed in the normal green leaf inbred line and the yellow-green leaf mutant: 536 genes were up-regulated, and 586 genes were down-regulated in the yellowgreen leaf mutant (Fig.4A). Among these differentially expressed genes, 1092 genes were categorized into 33 metabolic pathways (Fig.4B): 14 in photosynthesis, 19 in lipid metabolism, 40 in hormone metabolic, 53 in transport, 52 in secondary metabolism, and 55 in stress response, 106 in RNA synthesis, and another 106 in protein metabolism. The remaining 328 genes could not be mapped to specific metabolism pathways.

\section{Differentially expressed genes in chlorophyll biosynthetic pathways}

Chlorophylls are synthesized from the precursor protophorphyrin IX. As shown in Fig. 5, three chlorophyll metabolic genes (magnesium-chelatase subunit chID [Zm00001d013013], protochlorophyllide reductase $A$ [Zm00001d001820], and chlorophyllide a oxygenase [Zm00001d004531] showed decreased expression levels in the yellow-green leaf mutant. Similarly, two protophorphyrin biosynthetic genes (coproporphyrinogen III oxidase [Zm00001d026277] and uroporphyrinogen decarboxylase 1 [Zm00001d044321]) displayed decreased expression in the yellow-green leaf mutant. However, genes encoding chlorophyllide a oxygenase (Zm00001d002358) and protoporphyrinogen oxidase (Zm00001d 008203) showed increased transcript abundance in the yellow-green leaf mutant.

\section{Differentially expressed genes in photosynthetic reactions}

As shown in Fig.6, 22 genes in photosynthesis were differentially expressed in the yellow-green leaf mutant and the normal green leaf inbred line. Of these genes, two are related to photosynthetic light reactions, 11 genes participate in photorespiration, and nine are involved in the Calvin cycle. Among the two genes in photosynthetic light reactions, one encodes a Mog1/PsbP/DUF1795-like photosystem II reaction center PsbP family protein (Zm00001d041824) and the other encodes PGRL1A (PGR5-like protein 1A, Zm00001d034904). These two genes exhibited increased transcript abundance in the yellowgreen leaf mutant. However, genes encoding two ribulose bisphosphate carboxylase/oxygenase (RuBisCO) large chain precursors (Zm00001d00279 and GRMZM5G815453), which are involved in the Calvin cycle and photorespiration, showed decreased transcript abundance in the yellow-green leaf mutant. Genes encoding two RuBisCO large subunit-binding protein subunit alpha genes (Zm00001d031503 and Zm00001d051252) displayed decreased transcript abundance in the yellow-green leaf mutant as well. Genes encoding chloroplast chaperonin 60 subunit beta (Zm00001d035937), RuBisCO methyltransferase family protein (Zm00001d020437), TCP/cpn60 chaperonin family protein (Zm00001d045544), and RuBisCO large subunitbinding protein subunit alpha (Zm00001d00399) showed increased transcript abundancein the yellow-green leaf mutant. The expression of phosphoglycolate phosphatase (Zm00001d034887) and glycerate dehydrogenase (Zm00001d014919) was enhanced in the yellow-green leaf mutant. Whereas the expression of glycine dehydrogenase (Zm00001d023437) and aldolase superfamily protein (Zm00001d040084) was decreased.

\section{Differentially expressed genes in the tricarboxylic acid cycle}

The tricarboxylic acid cycle (TCA cycle) is responsible for the production of most of the ATP yield. As shown in Fig.7, a total of nine genes in the TCA cycle were down-regulated in the yellow-green leaf mutant. Among these genes, pyruvate phosphate dikinase (Zm00001d010321) catalyzes the conversion of pyruvate to phosphoenolpyruvate (PEP), aconitatehydratase (Zm00001d015497) catalyzes the stereo-specific isomerization of citrate to isocitrate, and isocitrate dehydrogense

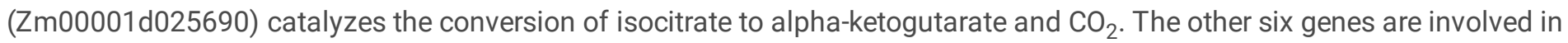
oxidative phosphorylation: NADH-ubiquinone oxidoreductase $20 \mathrm{kDa}$ subunit (Zm00001d043619), NADH dehydrogenase (Zm00001d016864), ubiquinol-cytochrome c reductase iron-sulfur subunit (Zm00001d016619), cytochrome c 
(Zm00001d042600), member of uncoupling protein PUMP2 family (Zm00001d048583), and cytochrome c oxidase (Zm00001d051055).

\section{Differentially expressed genes in the sucrose-to-starch pathway}

In Fig.8, nine genes in the sucrose-to-starch pathway were found to be differentially expressed between the yellow-green leaf mutant and the normal green leaf inbred line. Among these genes, two were up-regulated and seven were down-regulated in the yellow-green leaf mutant. Among the two up-regulated genes, one encodes aglycosyl hydrolase family 32 protein (Zm00001d025943), which may function as the invertase that split sucrose into glucose and fructose. The other gene is annotated as fructokinase-like protein (Zm00001d033181), which may catalyze the conversion of fructose to fructose-6phosphate. Among the seven down-regulated genes, three are annotated as granule-bound starch synthase $1 \mathrm{~b}$ (Zm00001d027242, Zm00001d029360, and Zm00001d019479), two encode soluble starch synthase (Zm00001d0002256 and Zm00001d0045261) and one encodes 1, 4-alpha-glucan branching enzyme IIB (Zm00001d003817). These six genes participate in starch synthesis. The other down-regulated gene is annotated as alpha-1, 4 glucanphosphrylase $L$ isozyme (Zm00001d034074), which catalyzes the conversion of starch to glucose-1-phosphate.

\section{Validation of unigenes and gene expression profiling}

To evaluate the validity of sequence data, nine known transcripts were selected for examination by real-time RT-PCR. Information for these genes and their gene-specific primers are showed in Supplementary Table 1. The results showed that the expression patterns determined using both RT-qPCR and DGE were consistent for nine genes, which suggesting that the transcriptome analyses were very reliable (Fig. 9).

\section{Discussion}

In maize, a yellow-green leaf mutant SN62 has been identified [16]. Photosynthetic characteristics of SN62 revealed that its chlorophyll content, the quantum efficiency of PSII and maximal quantum yield of PSII photochemistry were significantly lower than those of a medium-green leaf inbred line SN12[16]. In this study, another yellow-green leaf mutant was identified. The values of photosynthetic parameters in this newly identified yellow-green leaf mutant were comparable to those in the previously identified yellow-green leaf mutant (SN62). The chlorophyll content and the values of chlorophyll fluorescence parameters (Ft, Fo, Fm, Fo', Fm' and ETR) in the yellow-green leaf mutant were significantly lower than those in the normal green leaf inbred line. These data indicate that the yellow-green leaf mutant has fewer opened PSII reaction centers than the normal green leaf inbred line. However, the yellow-green leaf mutant and the normal green leaf inbred line had the similar value of the maximum photochemical quantum yield of PSII (Fv/Fm). This suggests that light absorption and energy transfer of the lightharvesting complexes is still efficient in the yellow-green leaf mutant plants. In addition, there were no obvious differences in

$F_{P S I l}, F_{N O}, F_{N P Q}, N P Q$, qN, qP, and qL between the yellow-green leaf mutant and the normal green leaf inbred line. Furthermore, Pn was only lower in the yellow-green leaf mutant than in the normal green leaf inbred line when the light intensity was at 1200 $\mu \mathrm{mol}$ photons $\mathrm{m}^{-2} \mathrm{~s}^{-1}$ or higher.

In seed plants, most of the genes responsible for the chlorophyll biosynthesis pathway have been identified [17]. Magnesiumprotoporphyrin chelatase catalyzes the first step in chlorophyll synthesis. This enzyme contains three subunits $(\mathrm{ChIH}, \mathrm{ChID}$ and ChII) and catalyzes the insertion of $\mathrm{Mg}^{2+}$ into protoporphyrin IX. Mutation in ChID resulted in a chlorina (yellowish-green) phenotype in rice [18]. Protochlorophyllide reductase catalyzes the conversion of pchlide to chloro-phyllide. Chlorophyllide $a$ oxygenase (CAO) is responsible for chlorophyll $b$ biosynthesis [19]. Overexpression of $C A O$ was found to enlarge the antenna size of photosystem II in Arabidopsis [20]. Mutation in the barley CAO (fch2) gene leads to chlorophyll $b$ deficiency, which may affect electron transfer in photosystem II [21]. Coproporphyrinogen III oxidase is a key enzyme in the biosynthetic pathway of chlorophyll and heme. The deficiency in coproporphyrinogen III oxidase caused lesion formation in Arabidopsis [22].

Uroporphyrinogen decarboxylaseis responsible for the decarboxylation of four acetate groups of uroporphyrinogen III to yield coproporphyrinogen III, resulting inheme and chlorophyll biosynthesis [23]. In this study, these genes were found to have 
decreased transcript abundance in the yellow-green leaf mutant, which may directly cause chlorophyll deficiency and reduced formation of light-harvesting antenna complexes.

Photosynthesis begins with the light reactions. In this work, the gene encoding Mog1/PsbP/DUF1795-like photosystem II reaction center PsbP family protein and the gene annotated as PGRL1A were found to have increased transcript abundance. $\mathrm{PsbP}$ is necessary for the retention of $\mathrm{Ca}^{2+}$ and $\mathrm{Cl}^{-1}$, the assembly of PSIl complex, and the maintenance of normal thylakoid architecture in PSII [24]. PGRL1A is associated with PSI and it interacts with PGRL1 [25]. The PGRL1-PGR5 complex was found to facilitate cyclic electron flow [25]. In this work, the expression of both genes was up-regulated in the yellow-green leaf mutant, which may make the fewer opened PSII reaction centers work efficiently in the yellow-green leaf mutant.

TCA cycle plays a central role in generating ATP and providing reducing agent NADH and precursors for a number of amino acids in both heterotrophic and photosynthetic tissues [26]. In this work, totally nine genes were identified with down-regulated expression in the yellow-green leaf mutant, including pyruvatephosphate dikinase, aconitatehydratase, isocitrate dehydrogenase, $\mathrm{NADH}$ dehydrogenase, and $\mathrm{NADH}$-ubiquinone oxidoreductase $20 \mathrm{kDa}$ subunit. Pyruvate phosphate dikinase is the key enzyme in cellular energy metabolism; it catalyzes the ATP- and phosphate $\left(\mathrm{P}_{\mathrm{i}}\right)$-dependent conversion of pyruvate to phosphoenol pyruvate in C4 plants [27]. Aconitate hydratase catalyzes the conversion of citrate to cis-aconitate [28]. Isocitrate dehydrogenase catalyzes oxidative decarboxylation of isocitrate [29]. The other six genes are involved in the mitochondrial electron transport chain and ATP synthesis, which requires the participation of large protein complex I (NADH-ubiquinone oxidoreductase), II (NADH dehydrogenase), III (ubiquinol-cytochrome $c$ reductase) and IV (cytochrome $c$ oxidase) [30, 31]. The decreased expression of these genes may have negative effects on ATP generation in the mitochondria of the yellow-green leaf mutant. Furthermore, seven genes involved in the conversion of sucrose to starch were found to have decreased transcript abundance in the yellow-green leaf mutant. Of these genes, granule-bound starch synthase $1 \mathrm{~b}$ is responsible for amylose synthesis [32]. Soluble starch synthase is a key enzyme in the biosynthesis of amylopectin [33]. Moreover, the analysis of enzymes activities in starch biosynthesis pathway further demonstrated the results. The enzymes activities of SS, SSS, SPS and GBSS were significantly lower in yellow-green leaf if compared with that in normal green leaf (Additional file: Fig. S2). But there were no obvious difference in the contents of starch and total reducing sugar, though the content of the water-soluble total sugar was high in normal green leaf (Additional file: Fig. S3).

\section{Conclusions}

In summary, a number of genes in chlorophyll biosynthesis, photosynthesis, tricarboxylic acid cycle, and sucrose-to-starch conversion were found to be differentially expressed in the yellow-green leaf mutant and the normal green leaf inbred line. These findings provide potential explanations for observed morphological and physiological changes in the yellow-green leaf mutant. Further investigations are needed to unravel the molecular basis of the morphological, physiological and transcriptional changes in the yellow-green leaf mutant plants.

\section{Methods}

\section{Plant material}

The maize inbred line $\mathrm{C} 033$ and LH102 were obtained from preserved breeds in rural areas of Anhui province which were stored in Tobacco Research Institute, Anhui province, People's Republic of China. The yellow-green leaf mutant inbred line was isolated from an F2 segregating population of the recombination between inbred line $\mathrm{C} 033$ and LH102 in the farm of Tobacco Research Institute. After successive self-pollination of F2, F3 and F4 generations, a stable F5 generation was obtained. The yellow-green leaf mutant inbred line and a normal green leaf inbred line from the F5 generation were selected for downstream analyses. The inbred lines were cultivated with regular water and fertilizer management in the farm of Tobacco Research Institute in the year of 2017. After the third leaf was fully expanded, seedlings were selected for physiological parameter determination, RNA extraction, and gene expression analysis.

\section{Measurement of chlorophyll contents}


Leaf samples $(100 \mathrm{mg})$ were cut into small pieces, and soaked in $10 \mathrm{~mL}$ of $80 \%$ acetone (acetone: water $=4: 1)$ at $4{ }^{\circ} \mathrm{C}$ in the dark for $24 \mathrm{~h}$. The supernatant was collected after centrifugation at $6000 \mathrm{rpm}$ for $10 \mathrm{~min}$. The absorbance was recorded at 663 and $645 \mathrm{~nm}$ on a UV-1800 spectrophotometer (Shimadzu Corporation, Japan). The concentrations of Chl $a$ and Chl $b$ were calculated using the method described by Arnon [34].

\section{Measurement of carotenoid compounds}

To analyze the carotenoid compounds, $200 \mathrm{mg}$ leaf samples were grinded into powder with $2 \mathrm{ml}$ absolute alcohol containing $1 \%$ butylated hydroxytoluene. After water bath for $5 \mathrm{~min}$ at $85^{\circ} \mathrm{C}, 40 \mathrm{ul} 80 \% \mathrm{KOH}$ and $1 \mathrm{ml} \mathrm{N}$-hexane were added into the extraction buffer followed by water bath and vortex. The supernatant were eventually collected and dried with nitrogen, then dissolved in $500 \mathrm{ul}$ acetonitrile solution containing $1 \%$ butylated hydroxytoluene, $25 \%$ methanol, and $5 \%$ dichloromethane for following analysis.

The Ultimate 3000 UHPLC system (Thermo Fisher Scientific, USA) was employed to quantitatively and qualitatively determine the components. Carotenoids were resolved and analyzed on a reverse phase YMC Carotenoid column $(250 * 4.6 \mathrm{~mm}, 5 \mathrm{um}$; YMC, Kyoto, Japan) set at a temperature of $40^{\circ} \mathrm{C}$ with the flow rate of $1 \mathrm{ml} / \mathrm{min}$. The solvent system consisted of solvent $A$ with methanol: methyl tert-butyl ether: water (81:15:4, by vol) and solvent B with methanol: methyl tert-butyl ether (6.5:93.5, by vol). The gradient program was set as follows: 2 min hold on $100 \%$ solvent $A$, followed a 1 minute linear gradient to $32.5 \%$ solvent $A$ and $67.5 \%$ solvent $B$, then 2 min hold on $100 \%$ solvent B, and 2 min hold on $100 \%$ solvent A lastly. Carotenoid compounds were detected at $450 \mathrm{~nm}$.

\section{Measurement of the total starch, total sugar and enzymes activities}

The contents of the total starch, the total soluble sugar and the total reducing sugar were measured using the methods described by Mccleary et al., Irigoyen et al. and Miller et al. [35-37]. The enzymes activities of SS (Sucrose synthase) and SPS (Sucrose phosphate synthase) were assayed according to Echeverria and Humphreys' method [38]. The enzymes activities of SSS, GBSS and AGP were determined using the methods described by wang et al. and Nakamura et al [39-40].

\section{Measurements of chlorophyll fluorescence parameters}

By using the PAM-2500 chlorophyll fluorometer (Walz, German), chlorophyll fluorescence parameters were determined for the leaves from the yellow-green leaf mutant inbred line and the normal green leaf inbred line. The procedure was described as follows. After the leaf was adapted in the dark with the dark adapting clip for 20 min, the slow kinetics of chlorophyll a fluorescence induction was triggered with a continuous mode to measure dark- and light-adapted parameters. The leaf was initially subjected to a measuring light of $95 \mu \mathrm{mol}$ photons $\mathrm{m}^{-2} \mathrm{~s}^{-1}$. After Fo was recorded, a saturating pulse of $2000 \mu \mathrm{mol}$ photons $\mathrm{m}^{-2} \mathrm{~s}^{-1}$ was automatically turned on, and Fm was measured accordingly. At that time, an actinic light of $145 \mu \mathrm{mol}$ photons $\mathrm{m}^{-2} \mathrm{~s}^{-1}$ was activated to simulate normal irradiance conditions. F, Fm' and Fo' were subsequently measured with saturating pulses every $20 \mathrm{~s}$. The parameters $\Phi_{\mathrm{PS} I}, \Phi_{\mathrm{NPQ}}, \Phi_{\mathrm{NO}}, \mathrm{NPQ}, \mathrm{qN}, \mathrm{qP}, \mathrm{qL}$ and ETR were derived from the final measurements obtained after a 10-min light adaptation.

The maximum photochemical quantum yield of PSII (Fv/Fm) was calculated according to Stefanov and Terashima [41]: Fv/Fm $=(\mathrm{Fm}-\mathrm{Fo}) / \mathrm{Fm}$.

The effective photochemical quantum yield of PSII ( $\left.\Phi_{\text {PSII }}\right)$, the quantum yield of non-regulated heat dissipation and fluorescence emission $\left(\Phi_{\mathrm{NO}}\right)$, and quantum yield of light-induced non-photochemical quenching $\left(\Phi_{\mathrm{NPQ}}\right)$ were calculated as described by Kramer et al. [42]: $\Phi_{\mathrm{PSII}}=\left(\mathrm{F}^{\prime} \mathrm{m}-\mathrm{F}\right) / \mathrm{F}^{\prime} \mathrm{m}, \mathrm{F}_{\mathrm{NO}}=1 /(\mathrm{NPQ}+1+\mathrm{qL}(\mathrm{Fm} / \mathrm{Fo}-1)), \Phi_{\mathrm{NPQ}}=1-\Phi_{\mathrm{PSII}}-(1 /(\mathrm{NPQ}+1+\mathrm{qL}(\mathrm{Fm} / \mathrm{Fo}$ $-1))$.

The coefficient of photochemical quenching (qL) was calculated as described by Kramer (2004): qL = qP×F'o/F.

The coefficients of photochemical quenching (qP), non-photochemical quenching (qN and NPQ) were calculated as described by Stefanov and Terashima (2008): qP = (F'm-F)/(F'm-Fo), qN = 1-(Fm- $\left.F^{\prime} m\right) /\left(F m-F^{\prime} o\right), N P Q=\left(F m-F^{\prime} m\right) / F^{\prime} m$.

Page $7 / 21$ 
The relative apparent photosynthetic electron transport rate $(E T R)$ was calculated using the equation: $E T R=$ $\Phi_{\mathrm{PSII}} \times \mathrm{PAR} \times 0.5 \times 0.84$.

\section{Measurement of photosynthesis parameters}

The net photosynthetic rate (Pn) was measured using a Li-6400XT portable photosynthesis system (Li-Cor Inc., USA) equipped with a 6400-02B chamber and a red-blue LED light source with intensities up to $2000 \mu \mathrm{mol}$ photons $\mathrm{m}^{-2} \mathrm{~s}^{-1}$ over an area of 6 $\mathrm{cm}^{2}$. The flow rate was adjusted to $500 \mu \mathrm{mol} \cdot \mathrm{s}^{-1}$ with the absolute $\mathrm{CO}_{2}$ concentration of $380 \mu \mathrm{molmol}^{-1}$ at $26^{\circ} \mathrm{C}$ inside the chamber. The light response curve of $\mathrm{Pn}$ was determined at nine photosynthetically active radiation (PAR) levels $(0,50,100$, $200,400,800,1200,1600$ and $2000 \mu \mathrm{mol}$ photons $\mathrm{m}^{-2} \mathrm{~s}^{-1}$ ).

\section{Total RNA extraction for RNA-Seq and qRT-PCR}

Total RNA from the yellow-green leaf mutant and the normal green leaf inbred line was extracted with the RNAprep Pure Plant Kit (Tiangen, China) using $200 \mathrm{mg}$ tissue homogenized in liquid nitrogen. The quality and concentration of extracted RNA were determined using NanoDrop 2000 (Thermo, USA).

\section{cDNA library construction and sequencing}

By using Illumina TruSeq ${ }^{\text {TM }}$ RNA Sample Preparation Kit (Illumina, San Diego, USA), the cDNA library was constructed. After the quality detection, the Illumina sequencing was carried out at Beijing Novogene Biological Information Technology Co. Ltd. (Beijing, China) (HTTP://www.novogene.cn/). The index-coded samples were clustered following the manufacturer's instructions using TruSeq PE Cluster Kit v3-cBot-HS (Illumina). After cluster generation, the library was sequenced on an Illumina Hiseq 2500 platform with 200 bp paired-end reads.

\section{Data filtering and assembly}

By removing reads from adaptor sequences, duplicated sequences, ploy- $\mathrm{N}$ (reads with unknown nucleotides) and low-quality reads, high-quality clean reads were obtained for downstream analyses. The cleaned sequences were mapped to the maize B73 reference genome (AGPv4)

(ftp://ftp.ncbi.nlm.nih.gov/genomes/genbank/plant/Zea_mays/latest_assembly_versions/GCA_000005005.6_B73_RefGen_v4). The final transcriptome assembly was generated using TopHat 2 as previously described [43, 44]. Transcript counts were normalized by calculating reads per kilobase million mapped reads (RPKM) as described by Mortazavi et al. [45].

\section{Identification of differential expressed genes}

According to standard digital gene expression (DGE) methods as described by Zhang et al. [46], the read counts were adjusted by using edge $\mathrm{R}$ program package through one scaling normalized factor. Differentially expressed genes were screened by using the DEGseq R package (1.12.0; TNLIST, Beijing, China). A P-value of 0.005 was used as the threshold for significant differential expression according to the Benjamini and Hochberg's method [47].

\section{Gene functional annotation and metabolic pathway analysis}

The functions of differentially expressed genes were annotated using Blast $X\left(E-v a l u e ~<10^{-5}\right.$ ) queries searched against three databases: NCBI non-redundant (Nr) protein database (ftp://ftp.ncbi.nih.gov/blast/db/), Pfam (annotated Protein family) (http://pfam.xfam.org/), and SwissProt protein database (https://web.expasy.org/docs/swiss-prot_guideline.html). By using the Blast2GO program, the genes were annotated according to molecular function, biological process, and cellular component ontology. Functionally annotated genes were assigned to different metabolic pathways based on the Kyoto Encyclopedia of Genes and Genomes Pathway database (KEGG http://www.genome.jp/kegg). The MapMan software (V3.6.0 RC1) was used to map the target genes to metabolic pathways and draw the profile frames as described by Kakumanu et al. [48].

\section{Additional File}


Table S1 Primers for qRT-PCR analysis

Table S2 The list of differentially expressed genes between yellow-green leaf and normal green leaf

Table S3 The GO enrichment result of differentially expressed genes between yellow-green leaf and normal green leaf

Table S4 The KEGG pathway enrichment result of differentially expressed genes between yellow-green leaf and normal green leaf

Fig. S1 The Pearson correlation between individual RNA samples from the yellow-green leaf mutant and the normal green leaf inbred line.

Fig. S2 The activities of enzymes SS, SSS, SPS, GBSS and AGP involved in starch biosynthesis pathway

Fig. S3 The contents of starch, total reducing sugar and the water-soluble total sugar

Fig. S4 The Blast2GO analysis of differentially expressed genes between the yellow-green leaf mutant and the normal green leaf inbred line.

\section{Abbreviations}

Fo: the minimum fluorescence yield; Fm: the maximum fluorescence yield; Ft: the real-time fluorescence yield; Fv/Fm: The maximum photochemical quantum yield of PSII; $\Phi_{\mathrm{PSII}}$ : the effective photochemical quantum yield of PSIl; $\Phi_{\mathrm{NO}}$ : the quantum yield of non-regulated heat dissipation and fluorescence emission; $\Phi_{\mathrm{NPQ}}$ : quantum yield of light-induced non-photochemical quenching; qL: the coefficient of photochemical quenching; qP: the coefficient of photochemical quenching; NPQ: the coefficient of non-photochemical quenching; qN: the coefficient of non-photochemical quenching; ETR: he relative apparent photosynthetic electron transport rate; $\mathrm{Pn}$ : The net photosynthetic rate; RuBisCO: ribulose bisphosphate carboxylase/oxygenase; TCA: the tricarboxylic acid cycle; PEP: phosphoenol pyruvate; Chl: chlorophyll; CAO: Chlorophyllide a oxygenase;

\section{Declarations}

\section{Acknowledgements}

Not applicable.

\section{Funding}

This work was supported by National Natural Science Foundation of China (31601240), Scientific and Technological Innovation team of Anhui Academy of Agricultural Sciences and Fellowship of visiting scholar program of Anhui Academy of Agricultural Sciences (TL, a visiting scholar at the University of Tennessee). The above funding to TL, HY and YZ was used for the performing of the experiment, preparation of the maize inbred lines, analysis and interpretation of data in writing the manuscript.

\section{Availability of data and materials}

The datasets generated and analyzed in this study are available from the corresponding author on reasonable request.

\section{Author Contributions}

TL and HY performed experiments, generated figures, and wrote the draft manuscript. YL helped with data interpretation and revision of the manuscript. Q Dand GL helped in performing experiments and preparation of the materials. FC helped with data interpretation and revision of the manuscript. YZ designed the plan and significantly revised the manuscript. All authors have read and approved the final manuscript. 


\section{Ethics approval and consent to participate}

Not applicable.

\section{Consent for publication}

Not applicable

\section{Competing Interests}

The authors declare that they have no competing interests.

\section{References}

1. Gitelson AA, Gritz Y, Merzlyak MN. Relationships between leaf chlorophyll content and spectral reflectance and algorithms for non-destructive chlorophyll assessment in higher plant leaves. J plant physiol. 2003; 160(3), 271-282

2. Curran PJ, Dungan JL, Gholz HL.Exploring the relationship between reflectance red edge and chlorophyll content in slash pine. Tree Physiol. 1990; 7: 33 -48

3. Filella I, Serrano I, Serra J, Peñuelas J. Evaluating wheat nitrogen status with canopy relfectance indices and discriminant analysis. Crop Sci. 1995; 35: 1400-1405

4. Wu ZM, Zhang X, He B, Diao LP, Sheng SL, Wang JL, Guo XP, Su N, Wang LF, Jiang L, Wang CM, Zhai HQ, Wan JM.A chlorophyll-deficient rice mutant with impaired chlorophyllide esterification in chlorophyll biosynthesis.Plant physiol. 2007; 145(1): 29-40.

5. Wang Y, Zheng W, Zheng W, Zhu J, Liu Z, Qin J, Li H. Physiological and transcriptomic analyses of a yellow-green mutant with high photosynthetic efficiency in wheat (Triticumaestivum L.).Funct Integr Genomics. 2018; 18(2): 175-194.

6. Nothnagel T, Straka P. Inheritance and mapping of a yellow leaf mutant of carrot (Daucuscarota).Plant Breeding. 2003; 122(4):339-342.

7. Sawers RJ, Viney J, Farmer PR, Bussey RR, Olsefski G, Anufrikova K, Neil Hunter C, Brutnell TP. The maize Oil yellow1 (Oy1) gene encodes the I subunit of magnesium chelatase.Plant Mol Biol. 2006; 60(1): 95-106.

8. Liu XP, Yang C, Han FQ, Fang ZY, Yang LM, Zhuang M, Lv HH, Liu YM, Li ZS, Zhang YY. Genetics and fine mapping of a yellow-green leaf gene (ygl-1) in cabbage (Brassica oleracea var. capitata L.).Mol Breeding. 2016; 36(6):82.

9. Dong H, Fei GL, Wu CY, Wu FQ, Sun YY, Chen MJ, Ren YL, Zhou KN, Cheng ZJ, Wang JL, Jiang L, Zhang X, Guo XP, Lei CL, Su N, Wang HY, Wan JM. A rice virescent-yellow leaf mutant reveals new insights into the role and assembly of plastid caseinolytic protease in higher plants.Plant Physiol.2013; 162(4):1867-1880.

10. Sakuraba Y, Rahman ML, Cho SH, Kim YS, Koh HJ, Yoo SC, Paek NC. The rice faded green leaf locus encodes protochlorophyllideoxido reductase B and is essential for chlorophyll synthesis under high light conditions. Plant J. 2013; 74(1):122-133.

11. Li C, Hu Y, Huang R, Ma X, Wang Y, Liao T, Zhong P, Xiao F, Sun C, Xu Z, Deng X, Wang P. Mutation of FdC2 gene encoding a ferredoxin-like protein with C-terminal extension causes yellow-green leaf phenotype in rice.Plant Sci. 2015; 238:127-134.

12. Ma X, Sun X, Li C, Huan R, Sun C, Wang Y, Xiao F, Wang Q, Chen P, Ma F, Zhang K, Wang P, Deng X. Map-based cloning and characterization of the novel yellow-green leaf gene ys83 in rice (Oryza sativa).Plant PhysiolBiochem.2017; 111: 1-9.

13. Zhang F, Luo X, Hu B, Wan Y, Xie J. YGL138(t), encoding a putative signal recognition particle $54 \mathrm{kDa}$ protein, is involved in chloroplast development of rice. Rice (NY) 2013; 6(1):7. https://doi.org/ 10.1186/1939-8433-6-7

14. Guan H, Xu X, He C, Liu C, Liu Q, Dong R, Liu T, Wang L. Fine mapping and candidate gene analysis of the Leaf-Color Gene ygl-1in maize. PLoS One 2016; 11(4): e0153962.

15. Wang P, Grimm B. Comparative analysis of light-harvesting antennae and state transition in chlorina and cpSRP mutants. Plant Physiol. 2016; 172(3):1519-1531. 
16. Zhong X M, Sun SF, Li FH, Wang J, Shi ZS. Photosynthesis of a yellow-green mutant line in maize.Photosynthetica. 2015; 53(4): 499-505.

17. Tripathy BC, Pattanayak GK. Chlorophyll biosynthesis in higher plants. InPhotosynthesis. Dordrecht: Springer; 2012. p. 6394.

18. Zhang H, Li J, Yoo JH, Yoo SC, Cho SH, Koh HJ, Seo HS, Paek NC. Rice Chlorina-1 and Chlorina-9 encode ChID and ChII subunits of Mg-chelatase, a key enzyme for chlorophyll synthesis and chloroplast development.Plant Mol Biol. 2006; 62(3): 325-337.

19. Reinbothe C, Bartsch S, Eggink LL, Hoober JK, Brusslan J, Andrade-Paz R, Monnet J, Reinbothe S. A role for chlorophyllideaoxygenase in the regulated import and stabilization of light-harvesting chlorophyll a/b proteins. Proc Natl Acad Sci USA. 2006; 103(12): 4777-4782.

20. Tanaka R, Koshino Y, Sawa S, Ishiguro S, Okada K, Tanaka A. Overexpression of chlorophyllide a oxygenase (CAO) enlarges the antenna size of photosystem II in Arabidopsis thaliana. Plant J. 2001; 26(4): 365-373.

21. Mueller AH, Dockter C, Gough SP, Lundqvist U, von Wettstein D, Hansson M. Characterization of mutations in barley fch2 encoding chlorophyllidea oxygenase. Plant Cell Physiol. 2012; 53(7): 1232-1246.

22. Ishikawa A, Okamoto $H$, Iwasaki Y, Asahi T.A deficiency of coproporphyrinogen III oxidase causes lesion formation in Arabidopsis. Plant J. 2001; 27(2): 89-99.

23. Fan J, Liu Q, Hao Q, Teng M, Niu L. Crystal structure of uroporphyrinogen decarboxylase from Bacillus subtilis. J Bacterial. 2007; 189(9): 3573-3580.

24. Cao P, XieY, Li M, Pan XW, Zhang HM, Zhao XL, Su XD, Cheng T, Chang W. Crystal structure analysis of extrinsic PsbP protein of photosystem II reveals a manganese-induced conformational change. Mol Plant. 2015; 8(4): 664-666.

25. DalCorso G, Pesaresi P, Masiero S, Aseeva E, Schünemann D, Finazzi G, Joliot P,Barbato R, Leister D. A complex containing PGRL1 and PGR5 is involved in the switch between linear and cyclic electron flow in Arabidopsis. Cell. 2008; 132(2): 273285.

26. Daloso DM, Müller K, Obata T, Florian A, Tohge T, Bottcher A, Riondet C, Bariat L, Carrari F, Nunes-Nesi A, Buchanan BB, Reichheld JP, Arujo WL, Fernie AR. Thioredoxin, a master regulator of the tricarboxylic acid cycle in plant mitochondria. Proc Natl Acad Sci USA. 2015; 112(11): E1392-E1400.

27. Ciupka D, Gohlke H. On the potential alternate binding change mechanism in a dimeric structure of Pyruvate Phosphate Dikinase. Sci Rep. 2017; 7(1): 8020.

28. Lichardusova L, Tatarkova Z, Calkovska A, Mokra D, Engler I, Racay P, Lehotsky J, Kaplan P. Proteomic analysis of mitochondrial proteins in the guinea pig heart following long-term normobarichyperoxia.Mol Cell Biochem. 2017; 434(1-2): $61-73$

29. Mhamdi A, Noctor G. Analysis of the roles of the arabidopsisperoxisomalisocitrate dehydrogenase in leaf metabolism and oxidative stress. Environ Exp Bot. 2015; 114:22-29.

30. Møller IM. A new dawn for plant mitochondrial NAD(P)H dehydrogenases. Trends Plant Sci. 2002; 7(6): 235-237

31. Dudkina NV, Eubel H, Keegstra W, Boekema EJ, Braun HP. Structure of a mitochondrial supercomplex formed by respiratorychain complexes I and III.Proc Natl Acad Sci USA. 2005; 102(9): 3225-3229

32. Suzuki Y, Arae T, Green PJ, Yamaguchi J, ChibaY. AtCCR4a and AtCCR4b are involved in determining the poly (A) length of granule-bound starch synthase 1 transcript and modulating sucrose and starch metabolism in Arabidopsis thaliana. Plant Cell Physiol. 2015; 56(5): 863-874.

33. Wang Y, Li Y, Zhang H, Zhai H, Liu Q, He S. A soluble starch synthase I gene, IbSSI, alters the content, composition, granule size and structure of starch in transgenic sweet potato.Sci Rep. 2017; 7(1)-2315.

34. Arnon DI. Copper enzymes in isolated chloroplasts. Polyphenoloxidase in Beta vulgaris.Plant Physiol. 1949; 24(1): 1

35. Mccleary BV, Giboson TS, Solah V, Mugford DC. Total starch measurement in cereal products: interlaboratory evaluation of a rapid enzymic test procedure. Cereal Chem. 1994; 71(5): 501-504. 
36. Irigoyen JJ, Emerich DW, Sanchez-Diaz M. Water stress induced changes in concentrations of proline and total soluble sugars in nodulated alfalfa (Medicago sativa) plants. Physiol Plant. 1992; 84:55-60.

37. Miller GL. Use of dinitrosalicylic acid reagent for determination of reducing sugar. Anal Chem, 1959; 31(3): 426-428.

38. Echeverria E, Humphreys T. Glucose control of sucrose synthase in the maize scutellum. Phytochem. 1985; 24(12):28512855

39. Wang F, Chen S, Cheng F, Liu Y, Zhang G. The differences in grain weight and quality within a rice (Oryza sativa L.) panicle as affected by panicle type and source-sink relation. J Agron Crop Sci, 2007; 193(1), 63-73.

40. Nakamura Y, Yuki Y, Park SY. Carbohydrate metabolism in the developing endo-sperm of rice grains. Plant Cell Physiol. 1989; 30:833-839.

41. Stefanov D, Terashima I. Non-photochemical loss in PSII in high- and low-light-grown leaves of Vicia faba quantified by several fluorescence parameters including $L_{N P}$, Fo/Fm', a novel parameter.Physiol Plantarum. 2008; 133(2): 327-338

42. Kramer DM, Johnson G, Kiirats O, Edwards GE. New fluorescence parameters for the determination of QA redox state and excitation energy fluxes.Photosynth Res.2004; 79(2): 209.

43. Schnable PS, Ware D, Fulton R, Stein JC, Wei F, Pasternak S, et al. The B73 maize genome. Sci. 2009; 326:1112-1115.

44. Kim DC, Pertea G, Trapnell C, Pimentel H, Kelley R, Salzberg SL.TopHat2: accurate alignment of transcriptomes in the presence of insertions, deletions and gene fusions. Genome Biol. 2013; 14:R36

45. Mortazavi A, Williams BA, McCue K, Schaeffer L, Wold B. Mapping and quantifying mammalian transcriptomes by RNASeq.Nat Methods. 2008; 5(7): 621.

46. Zhang N, Zhang HJ, Zhao B, Sun QQ, Cao YY, Li R, Wu XX, Weeda S, Li L, Ren S, Reiter RJ, Guo YD. The RNA-seq approach to discriminate gene expression profiles in response to melatonin on cucumber lateral root formation.J Pineal Res. 2014; 56:39-50

47. Benjamini Y, Hochberg Y. Controlling the false discovery rate: a practical and powerful approach to multiple testing.J R Stat Soc Series B Stat Methodo. 1995; 289-300

48. Kakumanu A, Ambavaram MM, Klumas C, Krishnan A, Batlang U, Myers E, Grene R, Pereira, A. Effects of drought on gene expression in maize reproductive and leaf meristem tissue revealed by RNA-Seq. Plant Physiol. 2012; 160:846-867

\section{Figures}




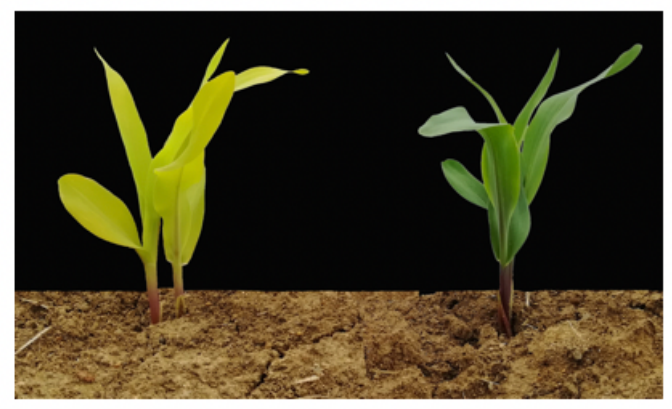

$\mathbf{A}$

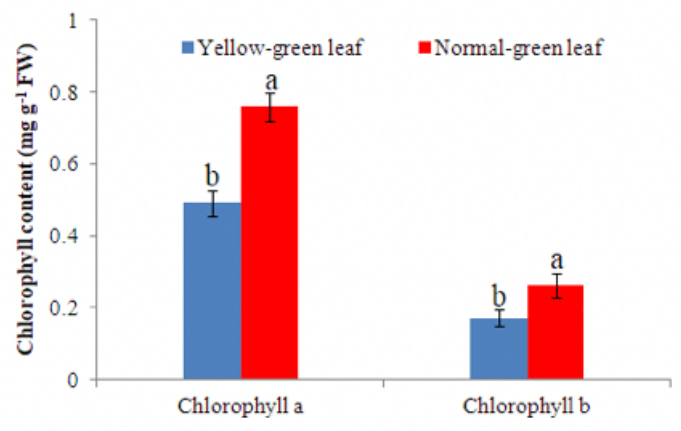

B

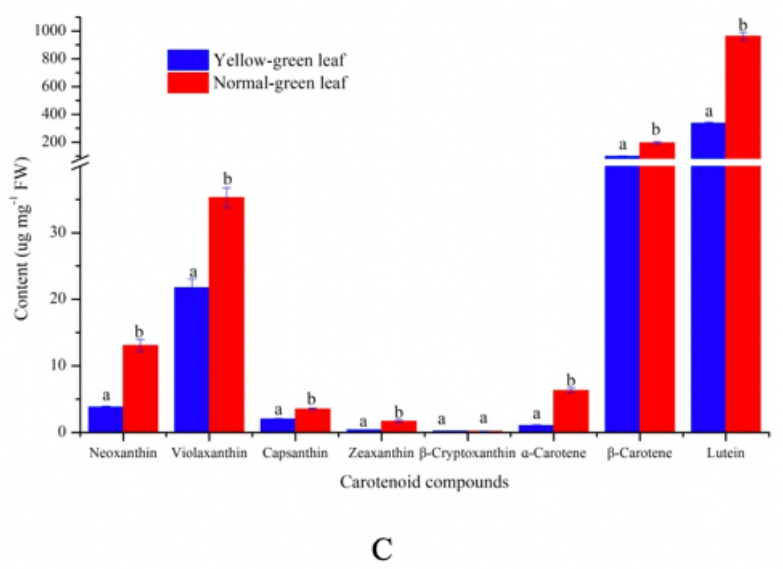

Figure 1

Phenotypic characteristics change of yellow-green leaf mutant plants. The letter A indicated two yellow-green leaf mutant plants (left) and a normal green leaf plant at the same age (right). Maize plants in this image were at the five-leaf stage. The letter B showed the contents of chlorophyll $a$ and chlorophyll $b$ in the normal green leaf inbred line and the yellow-green leaf mutant. The letter $\mathrm{C}$ showed the contents of eight carotenoid compounds including neoxanthin, violaxanthin, capsanthin, zeaxanthin, $\beta$-cryptoxanthin, 囚-carotene, $\beta$-carotene and lutein. Small letters $a$ and $b$ above the columns indicate differences between the yellow-green leaf mutant and the normal green leaf inbred line at $\mathrm{P}<0.05$, according to least significant difference (LSD) tests. FW is the abbreviation of the fresh weight. 


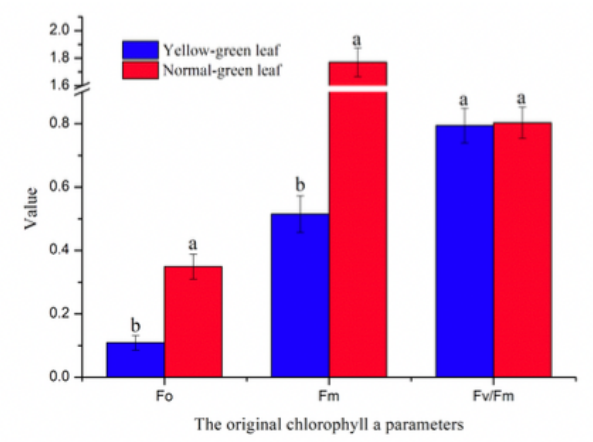

A

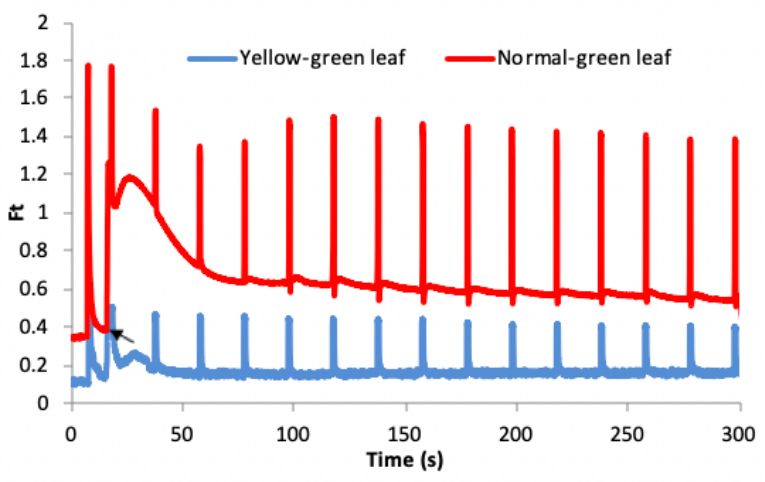

B

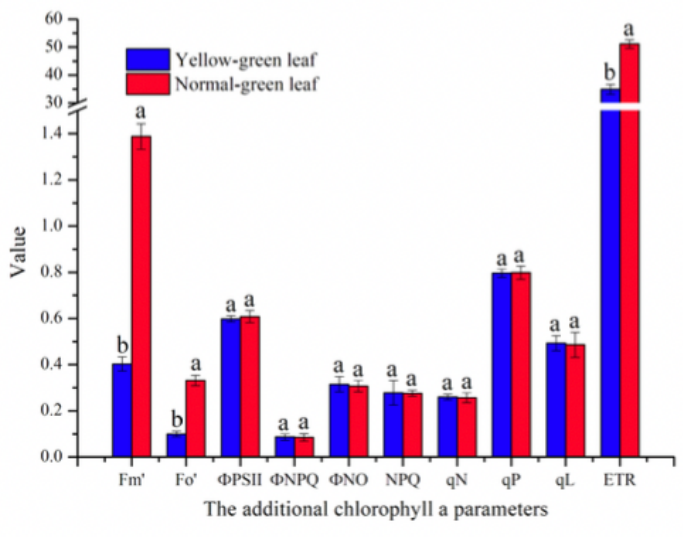

C

\section{Figure 2}

Chlorophyll fluorescence parameters in the yellow-green leaf mutant inbred line and the normal green leaf inbred line. The letter A indicated the original chlorophyll fluorescence parameters. The letter B showed real-time fluorescence yield Ft in the normal green leaf inbred line and the yellow-green leaf mutant. The point pointed by the arrows indicated the turned on of the actinic light. The letter $\mathrm{C}$ indicated additional chlorophyll fluorescence parameters of the normal green leaf inbred line and the yellowgreen leaf mutant. Small letters $a$ and $b$ indicate differences between the yellow-green leaf mutant and the normal green leaf inbred line at $\mathrm{P}<0.05$, according to least significant difference (LSD) tests. 


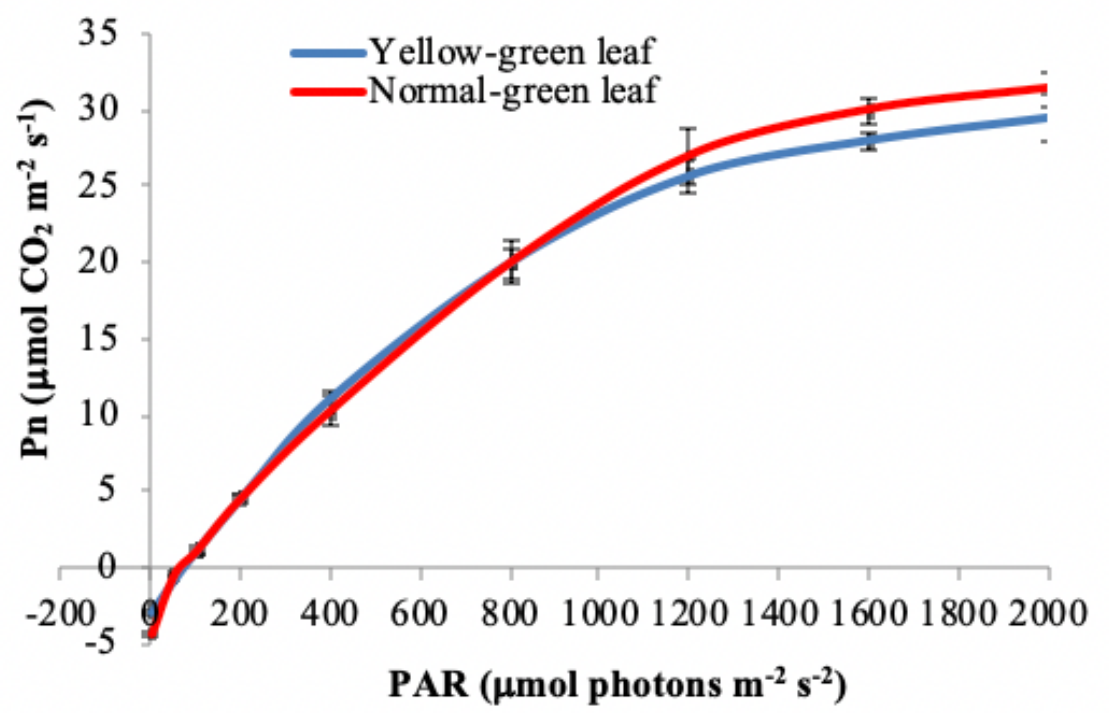

\section{Figure 3}

Light response curves of net photosynthesis in the yellow-green leaf mutant and the normal green leaf inbred line. Pn is the net photosynthesis rate. The light response curves were measured at nine PAR levels $(0,50,100,200,400,800,1200,1600$ and $2000 \mu \mathrm{mol}$ photons $\mathrm{m}-2 \mathrm{~s}-1)$. The dark respiration rate is defined as the Pn value when the light response curve intersects the Yaxis. The measurement was performed for three times. The error bars represented the standard errors. 

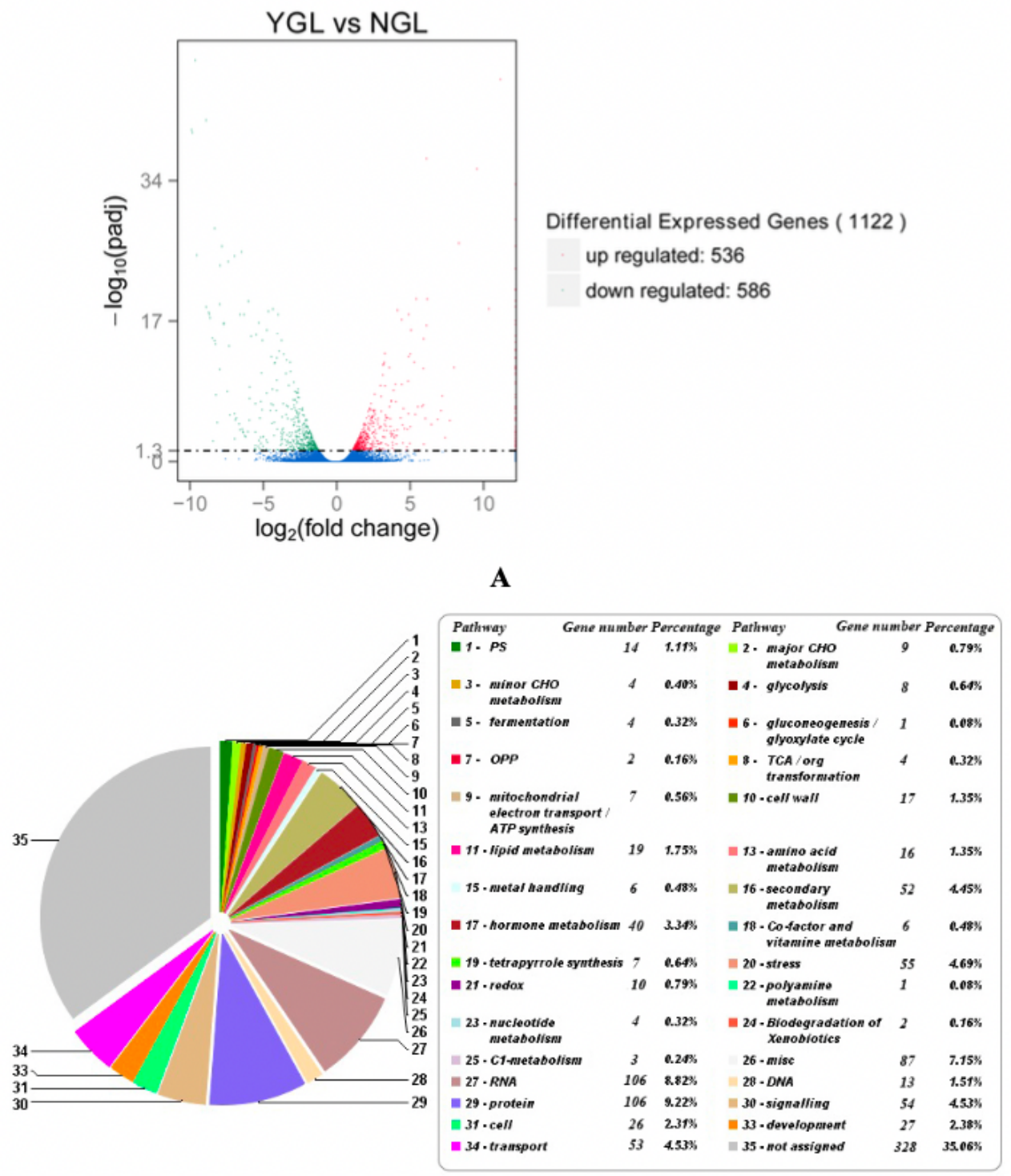

B

\section{Figure 4}

Differentially expressed genes and its enriched metabolic pathways between the yellow-green leaf mutant and the normal green leaf inbred line. The letter A indicated differentially expressed genes between the yellow-green leaf mutant and the normal green leaf inbred line. Differentially expressed genes were selected by q-value $<0.005 \& \mid \log 2$ (fold change) $\mid>1$. The $X$ axis indicates gene expression changes in different samples, and the $Y$ axis indicates the significant degree of gene expression changes. Scattered points represent each gene, the red dots represent differentially up-regulated genes, the green dots represent differentially down-regulated genes, and the blue dots represent no significant difference gene. YGL, yellow-green leaf mutant; NGL, normal green leaf inbred line; - $\log 10$ (padj), the corrected p-value (padj<0.05). The letter B showed the pie chart of enriched metabolic pathways of genes differentially expressed in the yellow-green leaf mutant and the normal green leaf inbred line. The pie chart was generated by submission of the differentially expressed genes to the online Mercator sequence annotation tool (http://www.plabipd.de/portal/mercator-sequence-annotation). 


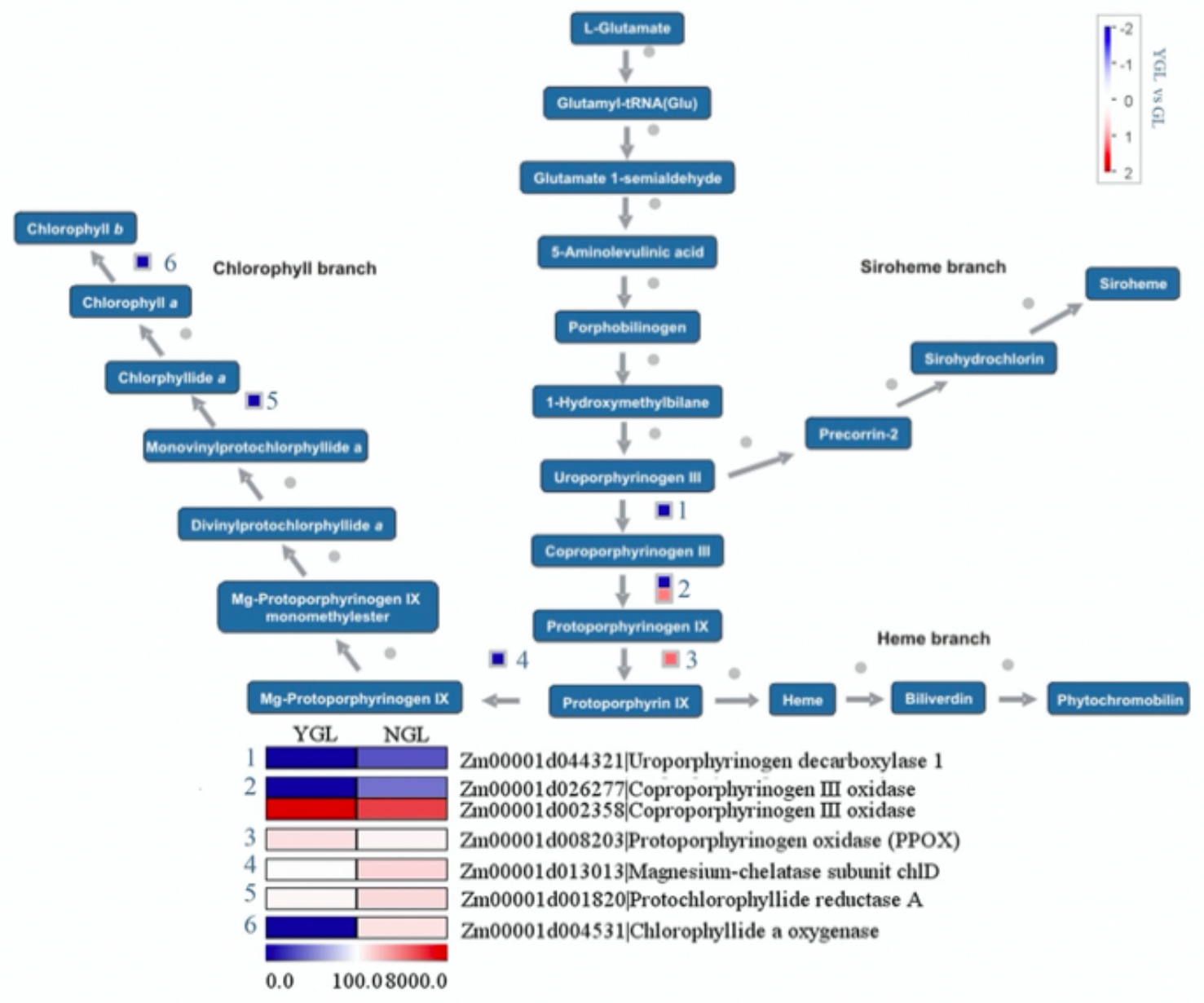

Figure 5

Comparative expression analysis of differentially expressed genes involved in chlorophyll metabolism between the yellow-green leaf mutant and the normal green leaf inbred line. 


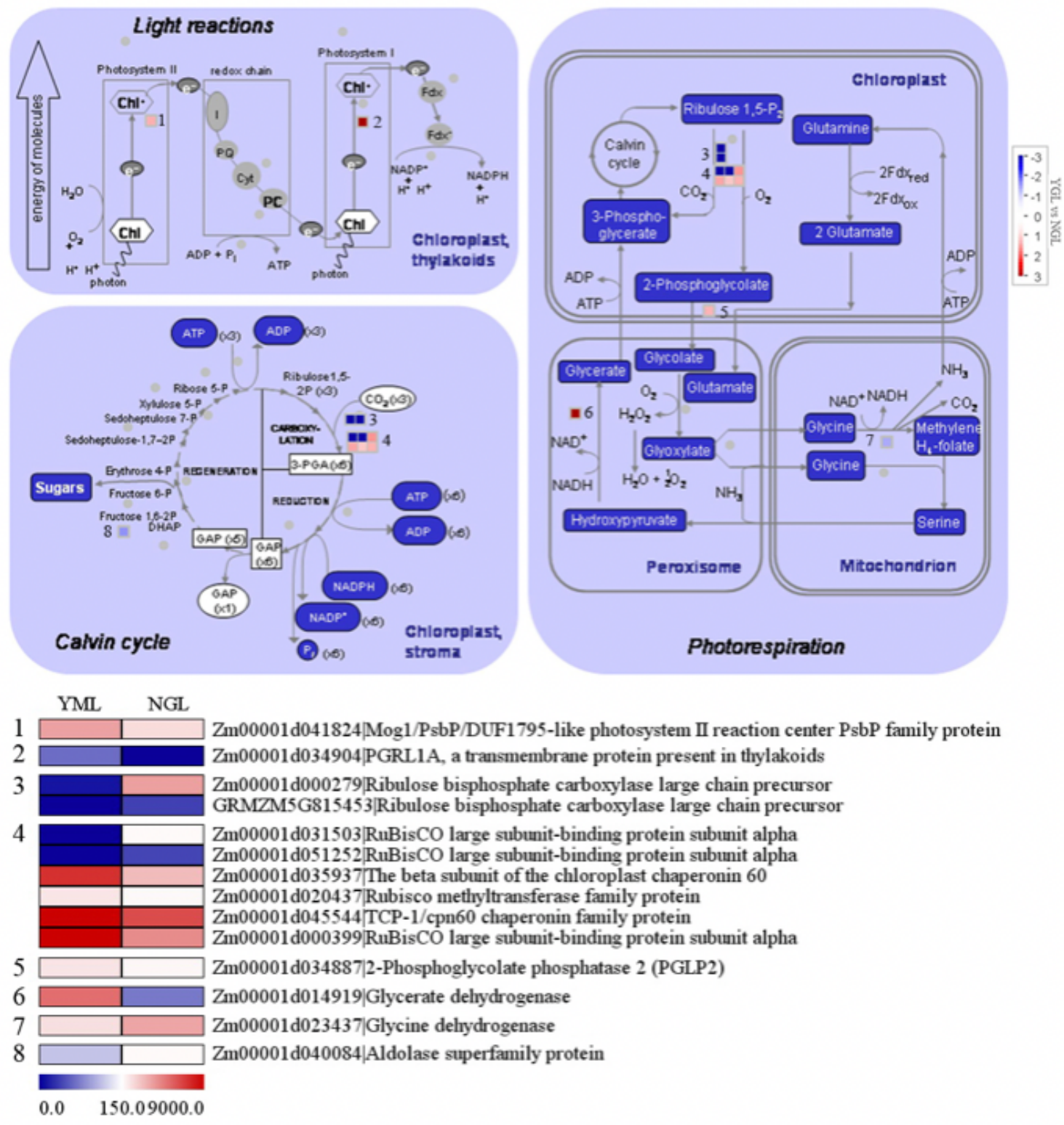

\section{Figure 6}

Comparative expression analysis of differentially expressed genes involved in photosynthesis light reactions and carbon reactions between the yellow-green leaf mutant and the normal green leaf inbred line. 


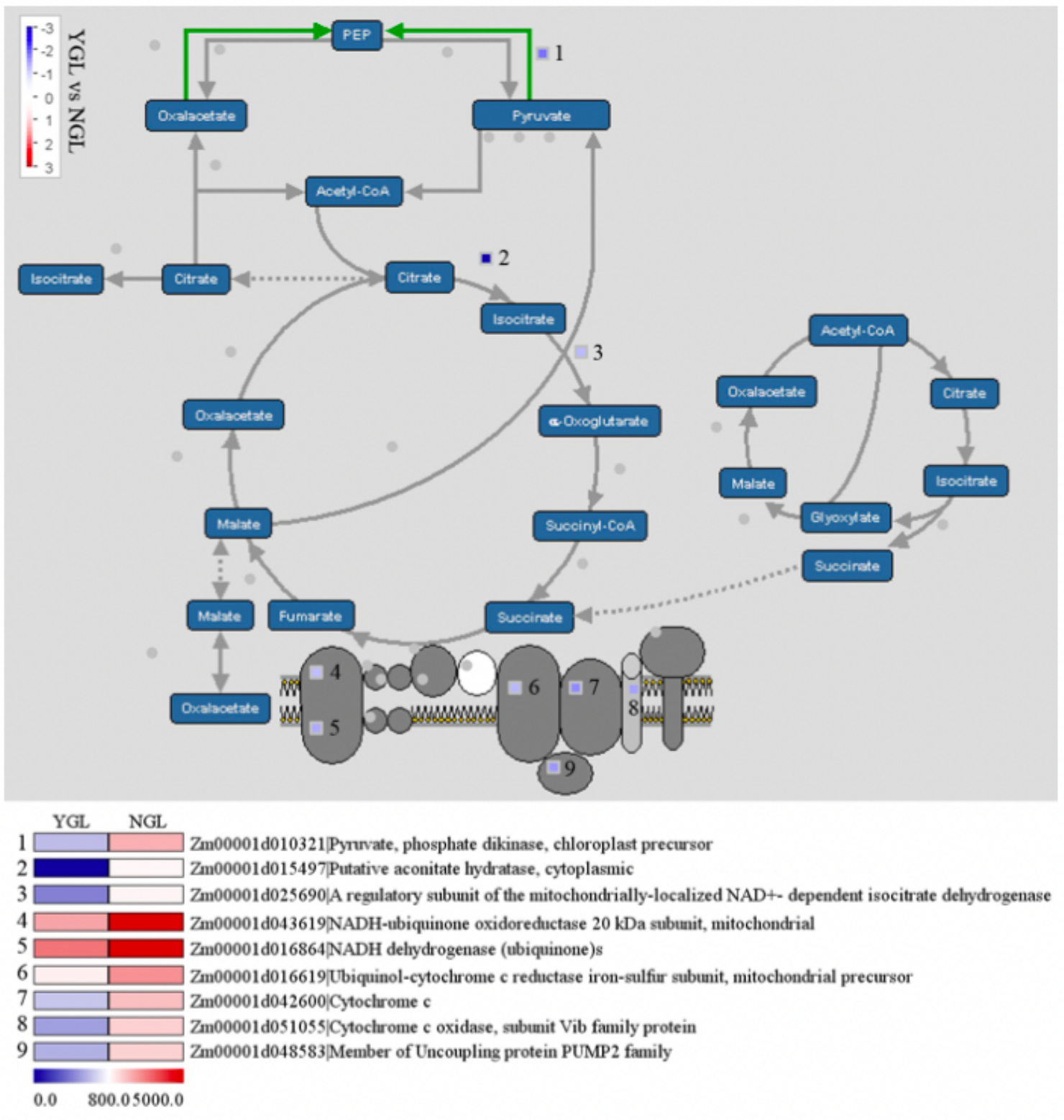

\section{Figure 7}

Comparative expression analysis of differentially expressed genes in the tricarboxylic acid cycle between the yellow-green leaf mutant and the normal green leaf inbred line. 


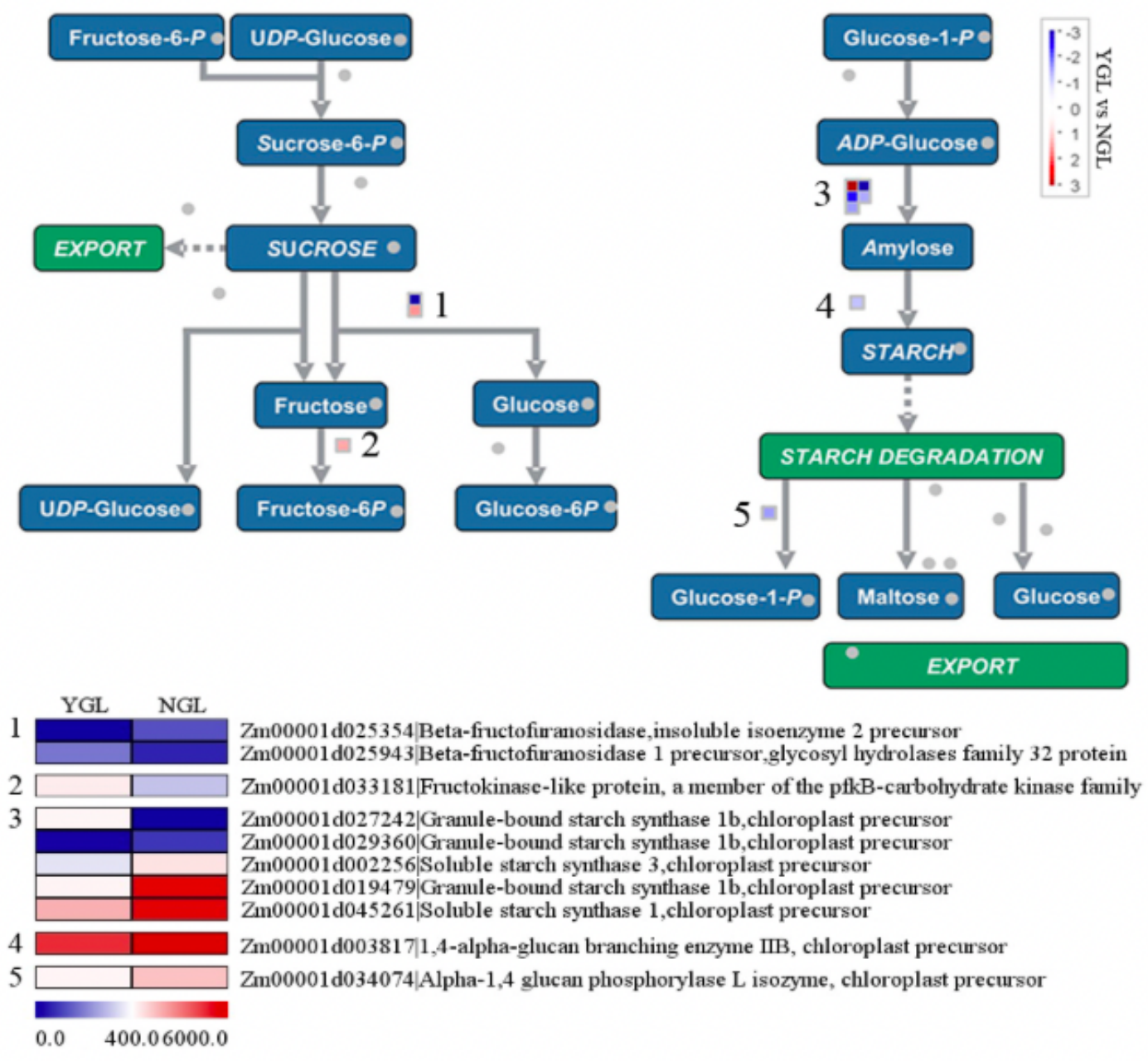

\section{Figure 8}

Comparative expression analysis of differentially expressed genes involved in sucrose to starch conversion between the yellowgreen leaf mutant and the normal green leaf inbred line. 


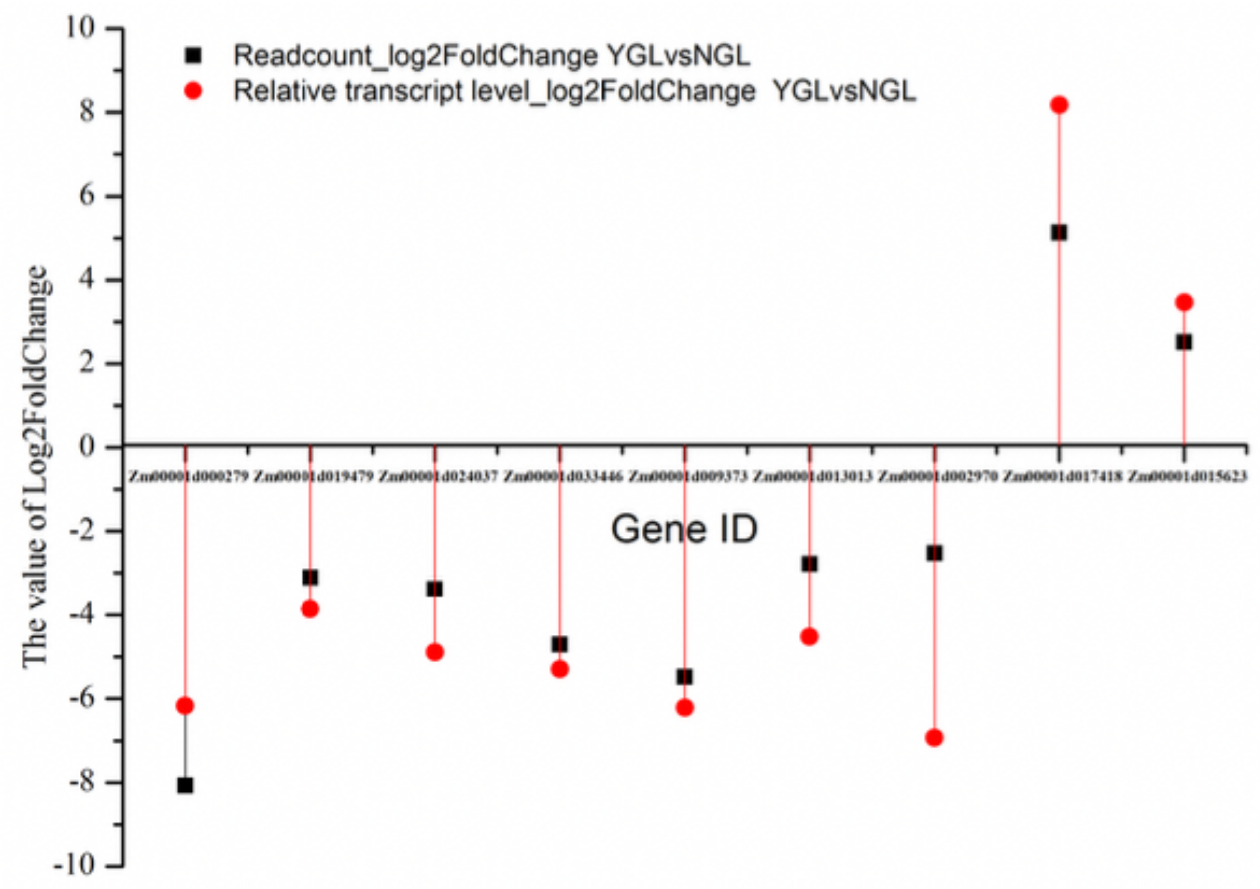

Figure 9

Validation of unigenes and DGE genes expression profiling

\section{Supplementary Files}

This is a list of supplementary files associated with this preprint. Click to download.

- Tables2.xls

- 3manuscriptSupplementarytableandfigures.docx

- Tables4.xls

- Tables3.xls 